Research Article

\title{
Structure and Stability of Steady State Bifurcation in a Cannibalism Model with Cross-Diffusion
}

\author{
Meijun Chen and Shengmao Fu \\ College of Mathematics and Statistics, Northwest Normal University, Lanzhou 730070, China \\ Correspondence should be addressed to Shengmao Fu; fusm@nwnu.edu.cn
}

Received 10 February 2020; Revised 10 June 2020; Accepted 25 June 2020; Published 21 July 2020

Academic Editor: Gen Q. Xu

Copyright (C) 2020 Meijun Chen and Shengmao Fu. This is an open access article distributed under the Creative Commons Attribution License, which permits unrestricted use, distribution, and reproduction in any medium, provided the original work is properly cited.

This paper deals with spatial patterns of a predator-prey crossdiffusion model with cannibalism. By applying the asymptotic analysis and Rabinowitz bifurcation theorem, we consider the local structure of steady state to the model and determine an explicit formula of the nonconstant steady state. Furthermore, the criteria of the stability/instability for the steady state with small amplitude are established.

\section{Introduction}

Multicomponent system is widely existed in nature and engineering apllications, for instance, in combustion, chemical reactors, tumor growth, gas mixtures, and animal crowds. On the diffusive level, these systems can be described by crossdiffusion equations taking into account multicomponent diffusion and reaction [1]. Specifically, crossdiffusion is a phenomenon in which the concentration gradient of one species induces a flux of the other species. The possibility of crossdiffusion terms in multicomponent systems was proposed by Onsager and Fuoss [2], while Baldwin et al. [3] undertook the experimental verification of the existence of crossdiffusion and also observed that the crossdiffusion coefficients can be quite significant. Since then, various crossdiffusion mathematical models have been suggested to interpret and predict many interesting features of natural multicomponent dynamics [4-10].

For example, Darcy's law implies that the velocity is proportional to the negative pressure gradient, and the pressure is defined by a state equation imposed by the volume extension of the mixture. Druet and Jungel [9] considered the convective transport in a multicomponent isothermal compressible fluid subject to the mass continuity equations. According to the idea that decomposes the system into a porous-medium-type equation for the volume extension and transport equations for the modified number fractions, they proved the global-in-time existence of classical and weak solutions in a bounded domain with nopenetration boundary conditions. Jungel and Ptashnyk [10] considered crossdiffusion systems defined in a heterogeneous medium, where the heterogeneity is reflected in spatially periodic diffusion coefficients or by the perforated domain. By combining two-scale convergence and the boundedness-by-entropy method, they proved two-scale homogenization limits of parabolic crossdiffusion systems in a heterogeneous medium with no-flux boundary conditions. Practical applications of such approach would investigate the problem of reducing a heterogeneous material to a homogeneous, specifically, predicting the response of refractory concrete to high-temperature exposure in steel furnaces. In nature, the impact of heat and mass transfer processes become particularly evident at high temperatures, where the increased pressure in pores, large temperature gradients, and temperature-induced creep may lead to catastrophic service failures [11]. Benes et al. [12] discussed a nonlinear numerical scheme arising from the implicit time discretization of the Bazant-Thonguthai model (with crossdiffusion) for hygrothermal behavior of concrete at high temperatures. By theoretical analysis and numerical simulation, they found that the model reproduces well the rapid increase of pore pressure in wet concrete due to 
extreme heating. In particular, there are three different zones which can be predicted: one corresponds to elements failed due to spalling damage, the other one indicates the part of the structure in which the local strength is sufficiently high to sustain the pore pressure, but its stability is lost due the explosive spalling of the former region, and the last one shows the portion of the cross-section is still capable of transmitting stresses due to mechanical loading, which is thereby responsible for the structural safety during fire. This phenomenon is particularly meaningful for the safety assessment of concrete structures prone to thermally induced spalling.

In fact, the rapid growth of the field of system biology has further contributed to interest in reaction-diffusion systems. Nowadays, crossdiffusion terms, which are inspired by the model, proposed by Shigesada et al. [13] in 1979 when they have studied the segregation of competing species, also have attracted wide attentions used in reaction-diffusion equations encountered in models from mathematical biology [14-21]. As we all know, the growth of biological population depends not only on time but also on spatial distribution. Spatial species interaction includes the self-diffusion and crossdiffusion. In this paper, we investigate a predator-prey system with both cannibalism and crossdiffusion in the form

$$
\begin{cases}u_{t}-d_{1} \Delta u=-u+v+a u v+u w, & x \in \Omega, t>0, \\ v_{t}-\Delta\left(d_{2} v+\frac{d_{4} v}{\varepsilon+u^{2}}\right)=b u-g v-u v, & x \in \Omega, t>0, \\ w_{t}-d_{3} \Delta w=s w-r w^{2}-e u w, & x \in \Omega, t>0, \\ \frac{\partial u}{\partial \nu}=\frac{\partial v}{\partial \nu}=\frac{\partial w}{\partial \nu}=0, & x \in \partial \Omega, t>0, \\ u(x, 0) \geq 0, v(x, 0) \geq 0, w(x, 0) \geq 0, & x \in \Omega,\end{cases}
$$

where $u(t), v(t)$, and $w(t)$ are the biomass of an adult predator, a juvenile predator, and prey at time $t$. $a, b, g, s, r, e$, and $\varepsilon$ are positive constants. $a$ denotes the cannibalism rate. The positive constants $d_{1}, d_{2}$, and $d_{3}$ are diffusion coefficients, and $d_{4}$ is the crossdiffusion coefficient. The initial values $u(x, 0), v(x, 0)$, and $w(x, 0)$ are nonnegative smooth functions which are not identically zero. $\Omega \subset \mathbb{R}^{N}$ is a bounded domain with smooth boundary $\partial \Omega$, and $v$ is the outward unit normal vector of the boundary $\partial \Omega$. The homogeneous Neumann boundary condition indicates that this system is self-contained with zero population flux across the boundary. For more details on the backgrounds of crossdiffusion models, one can see [21, 22].

The corresponding kinetic system of (1) was introduced by Magnsson in [23]. In this ODE system, Magnusson found that cannibalism, which is used as a means of population control to prevent them from overbreeding [21-31], has a destabilizing effect. When the large prey carries capacity, the juvenile mortality rate is high and adult recruitment rate is low, and stability of the equilibrium will be lost through Hopf bifurcation, the appearance of which is caused by the increasing level of cannibalism. And sustained oscillations can set in for sufficiently high levels of cannibalism. After, Kaewmanee and Tang [32] optimized the results of Magnusson, they obtained that stability of the equilibrium is lost due to the increase of the cannibalism attack rate past a bifurcation point that depends on other parameters. Later, the stability, topological properties, and types of bifurcations of the ODE system have been studied more explicitly by Marlk and Pribylova [33]. The authors proved that both subcritical and supercritical bifurcations may take place and hence the limits' cycle enclosing the stationary point does not need to be stable. Very recently, Zhang et al. [28] analyzed the effect of cannibalism and illustrated that the cannibalization rate can cause the local stability of the equilibrium changes from stable to unstable to stable, or from unstable to stable to unstable. Moreover, the positive equilibrium must be globally asymptotically stable if the cannibalization rate is large enough. They also obtained that supercritical/subcritical Hopf bifurcation can occur with the rates of two factors (the cannibalization and the benefit from cannibalism) as bifurcation parameters.

Recently, Fu and Yang [34] considered the corresponding pure diffusion system of $(1)\left(d_{4}=0\right)$ and proved that the positive equilibrium in this system has the same stability properties when it is regarded as equilibrium of the ODE system. It is shown that the decisive factor of destabilization for semilinear reaction-diffusion system is still cannibalism. Therefore, they further discussed the following strongly coupled crossdiffusion system (1).

Obviously, if $r \neq 0$ and

$$
\left\{\begin{array}{l}
b r+g s-g r>0, \\
s+e g+r(1-a b)>0, \\
s(1-a b)+e(g-b)>0,
\end{array}\right.
$$

then (1) has the unique positive equilibrium point $\tilde{u}=(\widetilde{u}, \widetilde{v}, \widetilde{w}), \quad$ where $\quad \tilde{u}=\left(\left(\alpha+\sqrt{\alpha^{2}+4 e \beta}\right) / 2 e\right)$, $\tilde{v}=(b \tilde{u} /(g+\tilde{u})), \widetilde{w}=((s-e \widetilde{u}) / r), \quad$ and $\quad \alpha=a b r+s-$ $r-e g, \beta=b r+g s-g r$. By the linearization analysis, Fu and Yang proved that positive equilibrium can undergo stability switch from stable in the ODE system and semilinear system to unstable in the crossdiffusion system if $d_{4}$ is sufficiently large. This means that the decisive factor of destabilization for the positive equilibrium point in (1) is the crossdiffusion rate, and cannibalism is an auxiliary destabilizing force. Besides, by using the Leray-Schauder degree theory, they also obtained the existence of nonconstant positive steady states. For completeness, we introduce this existence result, which can be proved by similar arguments as Theorem 5 in [21].

Theorem 1 (see [34]). Let $a, b, g, s, r, e, \varepsilon, d_{1}, d_{2}$, and $d_{3}$ be fixed positive constants such that $0<\varepsilon<\varepsilon_{0}, a g<1, r>s$, and $(A 1)$ hold. If $\tilde{\mu} \in\left(\mu_{n}, \mu_{n+1}\right)(n \geq 2)$ and the sum $\sum_{i=2}^{n} \operatorname{dim} E\left(\mu_{i}\right)$ is odd, then there exists a positive constant $d_{4}^{*}$ such that the steady state problem corresponding to (1) has at 
least one nonconstant positive solution for $d_{4} \geq d_{4}^{*}$. Here, let $\mu_{n}$ and $\mu_{n+1}$ be the eigenvalues of the operator $-\Delta$ on $\Omega$ with the homogeneous Neumann boundary condition.

Our main purpose in this paper is to describe in detail the local structure of the nonconstant steady states and discuss the stability and instability of bifurcation steady states.

The rest of the paper is organized as follows. In Section 2, the local bifurcation analysis is performed to examine the structure of bifurcating steady states. Furthermore, the stability and instability of bifurcating steady states with small amplitude will be given in Section 3; meanwhile, the paper ends with a brief discussion.

\section{Local Structure and Formula of Steady State Bifurcation}

The existence of nonconstant steady state of system (1) is established under the condition that $\tilde{\mu} \in\left(\mu_{n}, \mu_{n+1}\right)(n \geq 2)$ and the sum $\sum_{i=2}^{n} \operatorname{dim} E\left(\mu_{i}\right)$ is odd in [34]. In this section, using the asymptotic analysis and bifurcation theory similar to those in [35-38], we choose $d_{4}$ as a bifurcation parameter and fix the rest of the parameter to explore the local structure of nonconstant steady states of (1) bifurcating from the constant steady state $\widetilde{u}$ in one dimension.

Before proceeding, we present some properties about the negative Laplace operator. Let $0=\mu_{1}<\mu_{2}<\mu_{3}<\cdots$ be the eigenvalues of the operator $-\Delta$ on $\Omega$ with the homogeneous Neumann boundary condition, and let $E\left(\mu_{i}\right)$ be the eigenspace corresponding to $\mu_{i}$ in $H^{1}(\Omega)$. Let $\mathbf{X}$ be the closure of $\left[C^{1}(\bar{\Omega})\right]^{3}$ in $\left[H^{1}(\Omega)\right]^{3}, \quad\left\{\phi_{i j}: j=1,2, \ldots\right.$, $\left.\operatorname{dim} E\left(\mu_{i}\right)\right\}$ be an orthonormal basis of $E\left(\mu_{i}\right)$, and $\mathbf{X}_{i j}=\left\{\mathbf{c} \phi_{i j}: \mathbf{c} \in \mathbb{R}^{3}\right\}$. Then,

$$
\begin{aligned}
& \mathbf{X}=\oplus_{i=1}^{+\infty} \mathbf{X}_{i}, \\
& \mathbf{X}_{i}=\underset{j=1}{\oplus} \mathbf{X}_{i j} .
\end{aligned}
$$

In particular, for $\Omega=(0, l)$, it is well known that the problem

$$
\begin{cases}-\omega^{\prime \prime}(x)=\mu \omega(x), & x \in(0, l), \\ \omega^{\prime}(x)=0, & x=0, l,\end{cases}
$$

has a sequence of simple eigenvalues

$$
\mu_{j}=\left(\frac{\pi j}{l}\right)^{2}, \quad j=0,1,2, \ldots
$$

whose corresponding eigenfunctions are given by

$$
\omega_{j}(x)= \begin{cases}1, & j=0, \\ \cos \left(\frac{\pi j x}{l}\right), & j>0 .\end{cases}
$$

This set of eigenfunctions is an orthogonal basis in $L^{2}(0, l)$. For later use, we now define a Banach space $X$ by

$$
X=\left\{(u, v, w): u, v, w \in C^{2}([0, l]), u^{\prime}=v^{\prime}=w^{\prime}=0 \text { at } x=0, l\right\},
$$

equipped with usual $C^{2}$ norm, and a Hilbert space $Y$

$$
Y=L^{2}(0, l) \times L^{2}(0, l) \times L^{2}(0, l),
$$

with the inner product

$$
\begin{aligned}
\left(\rho_{1}, \rho_{2}, \rho_{3}\right)_{Y}= & \left(u_{1}, u_{2}, u_{3}\right)_{L^{2}(0, l)}+\left(v_{1}, v_{2}, v_{3}\right)_{L^{2}(0, l)} \\
& +\left(w_{1}, w_{2}, w_{3}\right)_{L^{2}(0, l)},
\end{aligned}
$$

for $\rho_{i}=\left(u_{i}, v_{i}, w_{i}\right), i=1,2,3$.

For the sake of simplify, we investigate the structure of nonconstant positive steady state of (1) in one-dimensional interval $\Omega=(0, l)$, i.e., consider the associated elliptic problem:

$$
\begin{cases}-d_{1} u^{\prime \prime}=-u+v+a u v+u w, & x \in(0, l), \\ -\left(d_{2} v+\frac{d_{4} v}{\varepsilon+u^{2}}\right)^{\prime \prime}=b u-g v-u v, & x \in(0, l), \\ -d_{3} w^{\prime \prime}=s w-r w^{2}-e u w, & x \in(0, l), \\ u^{\prime}=v^{\prime}=w^{\prime}=0, & x=0, l .\end{cases}
$$

Define the map $F:(0, \infty) \times X \longrightarrow Y$ as

$$
F\left(d_{4}, \mathbf{u}\right)=\left(\begin{array}{c}
d_{1} \Delta u-u+v+a u v+u w \\
\Delta\left(d_{2} v+\frac{d_{4} v}{\varepsilon+u^{2}}\right)+b u-g v-u v \\
d_{3} \Delta w+s w-r w^{2}-e u w
\end{array}\right) .
$$

Then, $\mathbf{u}=(u, v, w)$ is a solution of $(10)$, equivalent to it is a zero-point of the map F. Clearly,

$$
F\left(d_{4}, \tilde{u}\right)=0, \quad d_{4}>0 .
$$

Notice that

$$
\begin{aligned}
f(u, v) \equiv & \frac{v}{\varepsilon+u^{2}}=f(\widetilde{u}, \widetilde{u})+\left[f_{u}(\widetilde{u}, \widetilde{u})(u-\widetilde{u})\right. \\
& \left.+f_{v}(\widetilde{u}, \widetilde{u})(v-\widetilde{u})\right]+\cdots \\
= & \frac{\widetilde{u}}{\varepsilon+\widetilde{u}^{2}}-\frac{2 \widetilde{u}^{2}}{\left(\varepsilon+\widetilde{u}^{2}\right)^{2}}(u-\widetilde{u})+\frac{1}{\varepsilon+\tilde{u}^{2}}(v-\widetilde{u})+\cdots
\end{aligned}
$$

Assume

$$
d_{4}=d_{4}^{0}+\sum_{k=1}^{\infty} \tau^{k} d_{4}^{k}
$$

For nonconstant solution $\bar{u}_{s}=\left(u_{s}, v_{s}, w_{s}\right)$ of (10) bifurcating from $\tilde{u}$ with small amplitude, let 


$$
\begin{aligned}
& \left\{\begin{array}{l}
u_{s}=\tilde{u}+\sum_{k=1}^{\infty} \tau^{k} u_{k}, \\
v_{s}=\tilde{u}+\sum_{k=1}^{\infty} \tau^{k} v_{k}, \\
w_{s}=\widetilde{w}+\sum_{k=1}^{\infty} \tau^{k} w_{k},
\end{array}\right. \\
& \mathscr{L}_{3}(v, u, w):=\left(d_{2}+d_{4}^{0} h(\widetilde{u})\right) v^{\prime \prime}-\left(g+\widetilde{u}+\frac{d_{4}^{0} h^{\prime}(\widetilde{u}) \widetilde{v}(1+a \widetilde{u})}{d_{1}}\right) v \\
& +\left(b-\tilde{v}+\frac{d_{4}^{0} h^{\prime}(\widetilde{u}) \widetilde{v}^{2}}{d_{1} \tilde{u}}\right) u-\frac{d_{4}^{0} h^{\prime}(\widetilde{u}) \tilde{u} \widetilde{v}}{d_{1}} w
\end{aligned}
$$

where $0<\tau \ll 1$. Substituting (14) and (15) into (10) and equating the $O(\tau)$ and $O\left(\tau^{2}\right)$ terms, respectively, we derive two systems

$$
\begin{gathered}
\begin{cases}d_{1} u_{1}^{\prime \prime}+(-1+a \tilde{v}+\widetilde{w}) u_{1}+(1+a \tilde{u}) v_{1}+\tilde{u} w_{1}=0, & x \in(0, l), \\
\mathscr{L}_{3}\left(v_{1}, u_{1}, w_{1}\right)=0, & x \in(0, l), \\
d_{3} w_{1}^{\prime \prime}-e \widetilde{w} u_{1}-r \widetilde{w} w_{1}=0, & x \in(0, l), \\
u_{1}^{\prime}=v_{1}^{\prime}=w_{1}^{\prime}=0, & x=0, l,\end{cases} \\
\begin{cases}d_{1} u_{2}^{\prime \prime}+(-1+a \widetilde{v}+\widetilde{w}) u_{2}+(1+a \widetilde{u}) v_{2}+\widetilde{u} w_{2}=F_{1}, & x \in(0, l), \\
\mathscr{L}_{3}\left(v_{2}, u_{2}, w_{2}\right)=F_{2}, & x \in(0, l), \\
d_{3} w_{2}^{\prime \prime}-e \widetilde{w} u_{2}-r \tilde{w} w_{2}=F_{3}, & x \in(0, l), \\
u_{2}^{\prime}=v_{2}^{\prime}=w_{2}^{\prime}=0, & x=0, l,\end{cases}
\end{gathered}
$$

where

$$
\begin{aligned}
F_{1} & =-a u_{1} v_{1}-u_{1} w_{1}, \\
F_{3} & =r w_{1}^{2}+e u_{1} w_{1}, \\
h(\widetilde{u}) & =\frac{1}{\varepsilon+\widetilde{u}^{2}}, \\
h^{\prime}(\widetilde{u}) & =-\frac{2 \widetilde{u}}{\left(\varepsilon+\widetilde{u}^{2}\right)^{2}}, \\
h^{\prime \prime}(\widetilde{u}) & =\frac{2\left(3 \widetilde{u}^{2}-\varepsilon\right)}{\left(\varepsilon+\widetilde{u}^{2}\right)^{3}}, \\
F_{2} & =-d_{4}^{0}\left[\left(h^{\prime}(\widetilde{u}) u_{1} v_{1}\right)^{\prime \prime}+\left(h^{\prime \prime}(\widetilde{u}) \widetilde{v} u_{1} u_{1}^{\prime}\right)^{\prime}\right]-d_{4}^{1}\left[h(\widetilde{u}) v_{1}^{\prime \prime}+h^{\prime}(\widetilde{u}) \widetilde{v} u_{1}^{\prime \prime}\right]+u_{1} v_{1} .
\end{aligned}
$$


It is easy to get nonzero solutions for (unique up to a constant multiple for any given positive integer $j$, and this constant can be absorbed into $\tau$ in (15)) for (17) as

$$
\left\{\begin{array}{l}
u_{1}=\omega_{j}, \\
v_{1}=e_{1}(j) \omega_{j}, \\
e_{1}(j)=\frac{1}{1+a \tilde{u}}\left(\frac{\widetilde{v}}{\widetilde{u}}+\frac{e \tilde{u} \tilde{w}}{r \widetilde{w}+d_{3} \mu_{j}}+d_{1} \mu_{j}\right)>0, \\
w_{1}=h_{1}(j) \omega_{j}, \\
h_{1}(j)=-\frac{e \widetilde{w}}{r \widetilde{w}+d_{3} \mu_{j}}<0,
\end{array}\right.
$$

as long as $d_{4}^{0}$ is given by

$$
d_{4}^{0}=\frac{\left(g+\tilde{u}+d_{2} \mu_{j}\right) e_{1}-(b-\widetilde{v})}{-h(\widetilde{u}) \mu_{j} e_{1}-h^{\prime}(\tilde{u}) \widetilde{v} \mu_{j}}:=d_{4}^{0 j}, \quad j=1,2, \ldots .
$$

It is clearly known that $d_{4}^{0}>0$ if

$$
\begin{array}{r}
(g+\tilde{u}) \widetilde{v}<(b-\widetilde{v})(1+a \tilde{u}) \widetilde{u}, \\
e_{1}<\frac{2 \widetilde{u} \widetilde{v}}{\varepsilon+\widetilde{u}^{2}} .
\end{array}
$$

Here, in order to get the uniqueness of solution, we need assume that $d_{4}^{0 j} \neq d_{4}^{0 k}$ for any integer $j \neq k$.

Set

$$
d_{4 \min }=\min _{j \in \mathbb{Z}^{+}} d_{4}^{0 j}=\min _{j \in \mathbb{Z}^{+}}\left\{\frac{\left(g+\widetilde{u}+d_{2} \mu_{j}\right) e_{1}-(b-\widetilde{v})}{-h(\widetilde{u}) \mu_{j} e_{1}-h^{\prime}(\widetilde{u}) \widetilde{v} \mu_{j}}\right\}=d_{4}^{0 j_{0}},
$$

for a positive integer $j_{0}$. Since $d_{4}$ is regarded as the bifurcation parameter, $d_{4}^{0 j}, j=1,2, \ldots$, is called the possible bifurcation location for the formation of new patterns [38]. This means that the first bifurcation occurs when the parameter $d_{4}$ crosses the bifurcation value $d_{4 \min }$. If the bifurcation is stable, it will be the pattern $\left(u_{s}, v_{s}, w_{s}\right)$ with formulae given in (15) and (20).

In the following, we find the formula of $d_{4}^{1}$. The adjoint system of the homogeneous system associated with (18) is

$$
\begin{cases}d_{1} \bar{u}_{2}^{\prime \prime}+(-1+a \widetilde{v}+\widetilde{w}) \bar{u}_{2}+\left(b-\widetilde{v}+\frac{d_{4}^{0} h^{\prime}(\widetilde{u}) \widetilde{v}^{2}}{d_{1} \widetilde{u}}\right) \bar{v}_{2}-e \widetilde{w} \bar{w}_{2}=0, & x \in(0, l), \\ \left(d_{2}+d_{4}^{0} h(\widetilde{u})\right) \bar{v}_{2}^{\prime \prime}+(1+a \widetilde{u}) \bar{u}_{2}-\left(g+\widetilde{u}+\frac{d_{4}^{0} h^{\prime}(\widetilde{u}) \widetilde{v}(1+a \widetilde{u})}{d_{1}}\right) \bar{v}_{2}=0, & x \in(0, l), \\ d_{3} \bar{w}_{2}^{\prime \prime}+\widetilde{u} \bar{u}_{2}-\frac{d_{4}^{0} h^{\prime}(\widetilde{u}) \widetilde{u} \widetilde{v}_{1}}{d_{1}} \bar{v}_{2}-r \widetilde{w} \bar{w}_{2}=0, & x \in(0, l), \\ \bar{u}_{2}^{\prime}=\bar{v}_{2}^{\prime}=\bar{w}_{2}^{\prime}=0, & x=0, l,\end{cases}
$$

which has one solution

$$
\left\{\begin{array}{l}
\bar{u}_{2}=e_{2}(j) \omega_{j}, \\
e_{2}(j)=\frac{1}{1+a \tilde{u}}\left(d_{2} \mu_{j}+d_{4}^{0} h(\widetilde{u}) \mu_{j}+g+\widetilde{u}+\frac{d_{4}^{0} h^{\prime}(\tilde{u}) \widetilde{v}(1+a \tilde{u})}{d_{1}}\right) \\
\bar{v}_{2}=\omega_{j}, \\
\bar{w}_{2}=h_{2}(j) \omega_{j}, \\
h_{2}(j)=\frac{\tilde{u}}{\left(r \tilde{w}+d_{3} \mu_{j}\right)(1+a \tilde{u})}\left(d_{2} \mu_{j}+d_{4}^{0} h(\tilde{u}) \mu_{j}+g+\widetilde{u}\right)>0
\end{array}\right.
$$


where $\omega_{j}$ is defined as in (6) for $j=1,2, \ldots$. By the solvable condition for (18), that is, the vectors $\left(F_{1}, F_{2}, F_{3}\right)$ and $\left(\bar{u}_{2}, \bar{v}_{2}, \bar{w}_{2}\right)$ should be orthogonal in $L^{2}(0, l)$, we have the solvability equation for $d_{4}^{1}$ as follows:

$$
\int_{0}^{l}\left(\bar{u}_{2} F_{1}+\bar{v}_{2} F_{2}+\bar{w}_{2} F_{3}\right) \mathrm{d} x=0 .
$$

A direct computation gives

$$
d_{4}^{1}=d_{4}^{1 j}=0, \quad \text { for all } j=1,2, \ldots
$$

When $d_{4}^{1}=0$, for each $j, F_{2}$ in (19) can be simplified to $F_{2}=\frac{e_{1}}{2}+\left(2 d_{4}^{0} h^{\prime}(\widetilde{u}) e_{1} \mu_{j}+d_{4}^{0} h^{\prime \prime}(\widetilde{u}) \widetilde{v} \mu_{j}+\frac{e_{1}}{2}\right) \cos \left(2 \sqrt{\mu_{j}} x\right)$.
From this, a particular solution $\left(u_{2}, v_{2}, w_{2}\right)$ can be found as

$$
\left\{\begin{array}{l}
u_{2}=a_{1}(j)+a_{2}(j) \cos \left(2 \sqrt{\mu_{j}} x\right), \\
v_{2}=a_{3}(j)+a_{4}(j) \cos \left(2 \sqrt{\mu_{j}} x\right), \\
w_{2}=a_{5}(j)+a_{6}(j) \cos \left(2 \sqrt{\mu_{j}} x\right),
\end{array}\right.
$$

$$
\begin{aligned}
& A=-1+a \widetilde{v}+\widetilde{w} \\
& B=1+a \widetilde{u}, \\
& C=d_{2}+d_{4}^{0} h(\widetilde{u}) \\
& D=b-\widetilde{v}+\frac{d_{4}^{0} h^{\prime}(\widetilde{u}) \widetilde{v}^{2}}{d_{1} \tilde{u}}, \\
& E=g+\widetilde{u}+\frac{d_{4}^{0} h^{\prime}(\widetilde{u}) \widetilde{v}(1+a \widetilde{u})}{d_{1}}, \\
& F=\frac{d_{4}^{0} h^{\prime}(\widetilde{u}) \widetilde{u} \widetilde{v}}{d_{1}} \\
& G=2 d_{4}^{0} h^{\prime}(\widetilde{u}) e_{1} \mu_{j}+d_{4}^{0} h^{\prime \prime}(\widetilde{u}) \widetilde{v} \mu_{j}+\frac{e_{1}}{2}, \\
& N_{1}=(\widetilde{u} E-B F) r h_{1}^{2}+(e \widetilde{u} E-e B F-r \widetilde{w} E) h_{1}+e_{1} \widetilde{w} r(B-a E), \\
& M_{1}=2 \widetilde{w}[(A E+B D) r+(B F-\widetilde{u} E) e], \\
& M_{2}=64 d_{1} d_{3} C \mu_{j}^{3}+16\left(r \widetilde{w} d_{1} C-d_{3} A C+d_{1} d_{3} E\right) \mu_{j}^{2} \\
& +4\left(-d_{3} B D-d_{3} A E-r \widetilde{w} A C+r \widetilde{w} d_{1} E+e \widetilde{u} \widetilde{w} C\right) \mu \\
& -r \widetilde{w}(A E+B D)+(\widetilde{u} E-F B) e \widetilde{w}, \\
& N_{2}=16 d_{3} C\left(a e_{1}+h_{1}\right) \mu_{j}^{2}+4\left[-r \tilde{u} h_{1}^{2} C+\left(-e \tilde{u} C+r \tilde{w} C+d_{3} E\right) h_{1}\right. \\
& \left.+a e_{1}\left(\widetilde{w} r C+d_{3} E\right)-2 d_{3} G B\right] \mu_{j}+(B F-\widetilde{u} E) r h_{1}^{2} \\
& +(e B F-e \tilde{u} E+r \widetilde{w} E) h_{1}+\left(a e_{1} E-2 G B\right) r \widetilde{w}, \\
& a_{1}(j)=\frac{N_{1}}{M_{1}}, \\
& a_{2}(j)=-\frac{N_{2}}{2 M_{2}},
\end{aligned}
$$




$$
\begin{aligned}
a_{3}(j) & =-\frac{1}{B}\left(\left(A-\frac{\tilde{u} e}{r}\right) a_{1}-\frac{\tilde{u}\left(r h_{1}^{2}+e h_{1}\right)}{2 r \widetilde{w}}+\frac{a e_{1}+h_{1}}{2}\right), \\
a_{4} & =\frac{1}{B}\left(\left(4 d_{1} \mu_{j}-A+\frac{e \tilde{u} \widetilde{w}}{4 d_{1} \mu_{j}+r \widetilde{w}}\right) a_{2}+\frac{\left(r h_{1}^{2}+e h_{1}\right) \tilde{u}}{2\left(4 d_{1} \mu_{j}+r \tilde{w}\right)}-\frac{a e_{1}+h_{1}}{2}\right), \\
a_{5} & =-\frac{e}{r} a_{1}-\frac{r h_{1}^{2}+e h_{1}}{2 r \widetilde{w}} \\
a_{6} & =-\frac{e \widetilde{w} a_{2}}{4 d_{3} \mu_{j}+r \widetilde{w}}-\frac{r h_{1}^{2}+e h_{1}}{2\left(4 d_{3} \mu_{j}+r \tilde{w}\right)} .
\end{aligned}
$$

Since $d_{4}^{1}=0$, it is necessary for further analysis to obtain $d_{4}^{2}$. Following the process of getting (17) and (18), further computation up to the order $O\left(\tau^{3}\right)$ and equating the $O\left(\tau^{3}\right)$ term yields

$$
\begin{cases}d_{1} u_{3}^{\prime \prime}+(-1+a \widetilde{v}+\widetilde{w}) u_{3}+(1+a \widetilde{u}) v_{3}+\widetilde{u} w_{3}=F_{4}, & x \in(0, l), \\ \mathscr{L}_{3}\left(v_{3}, u_{3}, w_{3}\right)=F_{5}, & x \in(0, l), \\ d_{3} w_{3}^{\prime \prime}-e \widetilde{w} u_{3}-r \tilde{w} w_{3}=F_{6}, & x \in(0, l), \\ u_{2}^{\prime}=v_{2}^{\prime}=w_{2}^{\prime}=0, & x=0, l,\end{cases}
$$

where

$$
\begin{aligned}
F_{4}= & -a u_{1} v_{2}-a v_{1} u_{2}-u_{1} w_{2}-w_{1} u_{2}, \\
F_{6}= & 2 r w_{1} w_{2}+e u_{1} w_{2}+e u_{2} w_{1}, \\
h^{\prime \prime \prime}(\widetilde{u})= & -\frac{24\left(\widetilde{u}^{3}-\widetilde{u} \varepsilon\right)}{\left(\varepsilon+\widetilde{u}^{2}\right)^{4}}, \\
F_{5}= & -d_{4}^{0}\left\{h^{\prime}(\widetilde{u})\left(u_{1} v_{2}+u_{2} v_{1}\right)^{\prime \prime}+h^{\prime \prime}(\widetilde{u})\left(\left(\widetilde{v} u_{1} u_{2}\right)^{\prime \prime}+\left(\frac{1}{2} u_{1}^{2} v_{1}^{\prime}+u_{1} v_{1} u_{1}^{\prime}\right)^{\prime}\right)+\frac{1}{2} h^{\prime \prime \prime}(\widetilde{u}) \widetilde{v}\left(u_{1}^{2} u_{1}^{\prime}\right)^{\prime}\right\} \\
& -d_{4}^{2}\left\{h(\widetilde{u}) v_{1}^{\prime \prime}+h^{\prime}(\widetilde{u}) \widetilde{v} u_{1}^{\prime \prime}\right\}+u_{1} v_{2}+u_{2} v_{1} .
\end{aligned}
$$

The solvability condition can be simplified as Substituting (32) into (33) leads to

$\int_{0}^{l}\left(F_{4} e_{2} \cos \left(\frac{\pi j x}{l}\right)+F_{5} \cos \left(\frac{\pi j x}{l}\right)+F_{6} h_{2} \cos \left(\frac{\pi j x}{l}\right)\right) \mathrm{d} x=0$.

$$
\begin{aligned}
d_{4}^{2 j}= & \frac{-d_{4}^{0 j}}{4\left(h(\widetilde{u}) e_{1}+h^{\prime}(\widetilde{u}) \widetilde{u}\right)}\left\{2 h ^ { \prime } ( \widetilde { u } ) \left[\left[2 e_{1} a_{1}(j)+e_{1} a_{2}(j)+2 a_{3}(j)+a_{4}(j)\right]\right.\right. \\
& \left.+h^{\prime \prime}(\widetilde{u})\left(4 a_{1}(j)+2 a_{2}(j)+3 e_{1}\right)+2 h^{\prime \prime \prime}(\tilde{u}) \widetilde{u}\right\} \\
& +\frac{1}{2\left(h(\widetilde{u}) e_{1}+h^{\prime}(\widetilde{u}) \tilde{v}\right) \mu_{j}}\left\{e _ { 2 } \left[\left(a e_{1}+h_{1}\right)\left(2 a_{1}(j)+a_{2}(j)\right)+a\left(2 a_{3}+a_{4}\right)\right.\right.
\end{aligned}
$$




$$
\begin{aligned}
& \left.+2 a_{5}(j)+a_{6}(j)\right]-\left[2 a_{3}(j)+a_{4}(j)+e_{1}\left(2 a_{1}(j)+a_{2}(j)\right)\right] \\
& \left.-h_{2}\left[\left(2 a_{5}(j)+a_{6}(j)\right)\left(2 r h_{1}+e\right)+e h_{1}\left(2 a_{1}(j)+a_{2}(j)\right)\right]\right\} .
\end{aligned}
$$

According to the above computation and bifurcation theorem [38], the local bifurcation of (10) is given as follows.

Theorem 2. If for any positive integer $j \neq k, d_{4}^{0 j} \neq d_{4}^{0 k}$, then $d_{4}^{0 j}$ is a bifurcation value of the equation $F\left(d_{4}, \mathbf{u}\right)=0$ with respect to the curve $\left(d_{4}, \widetilde{u}\right), d_{4}>0$. Moreover, there is a oneparameter family of nontrivial solutions $\Gamma(\tau)=\left(d_{4}(\tau), u^{*}(\tau), v^{*}(\tau), w^{*}(\tau)\right)$ of $(10)$ for $|\tau|$ sufficiently small, where $d_{4}(\tau), u^{*}(\tau), v^{*}(\tau)$, and $w^{*}(\tau)$ are continuous functions such that $d_{4}^{0}=d_{4}^{0 j}$ and

$$
\begin{aligned}
& u^{*}(\tau)=\widetilde{u}+\tau u_{1}+\tau^{2} u_{2}+o\left(\tau^{2}\right), \\
& v^{*}(\tau)=\widetilde{u}+\tau v_{1}+\tau^{2} v_{2}+o\left(\tau^{2}\right), \\
& w^{*}(\tau)=\widetilde{w}+\tau w_{2}+\tau^{2} w_{2}+o\left(\tau^{2}\right) .
\end{aligned}
$$

The zero-point set of $F\left(d_{4}, \mathbf{u}\right)$ constitutes two curves $\left(d_{4}, \widetilde{u}\right)$ and $\Gamma(\tau)$ in a neighborhood of the bifurcation point $\left(d_{4}^{0 j}, \tilde{u}\right)$.

To clarify the relationship between the solution $\left(u_{s}, v_{s}, w_{s}\right)$ and its bifurcation location $d_{4}^{0 j}$, we may relabel $\left(u_{s}, v_{s}, w_{s}\right)$ as $\left(u_{s j}, v_{s j}, w_{s j}\right)$, i.e.,

$$
\left\{\begin{array}{l}
u_{s j}=\tilde{u}+\tau u_{1}+\tau^{2} u_{2}+\cdots+\tau^{k} u_{k}+\cdots, \\
v_{s j}=\widetilde{v}+\tau v_{1}+\tau^{2} v_{2}+\cdots+\tau^{k} v_{k}+\cdots \\
w_{s j}=\widetilde{w}+\tau w_{1}+\tau^{2} w_{2}+\cdots+\tau^{k} w_{k}+\cdots
\end{array}\right.
$$

As specified in [27], $(\widetilde{u}, \widetilde{v}, \widetilde{w})$ is called the base term of the pattern, and the pattern shape and its amplitude are primarily determined by the leading term $\left(u_{1}, v_{1}, w_{1}\right)$ when $\tau$ is small.

\section{Stability of Steady State Bifurcation}

This section is devoted to study the stability of the pattern solution $\left(u_{s j}, v_{s j}, w_{s j}\right)$ bifurcated from $\left(d_{4}^{0 j}, \tilde{u}\right)$ by analyzing the sign of the principal eigenvalue.

For (10), set

$$
\left\{\begin{array}{l}
u=u_{s j}+\varphi e^{\lambda t} \\
v=v_{s j}+\xi e^{\lambda t} \\
w=w_{s j}+\eta e^{\lambda t}
\end{array}\right.
$$

Then,

$$
\begin{cases}d_{1} \varphi^{\prime \prime}+\left(-1+a v_{s j}+w_{s j}\right) \varphi+\left(1+a u_{s j}\right) \xi+u_{s j} \eta=\lambda \varphi, & x \in(0, l), \\ \left(d_{2}+d_{4} h\left(u_{s j}\right)\right) \xi^{\prime \prime}+\mathscr{H} \varphi^{\prime \prime}+\mathscr{H} \xi^{\prime}+\mathscr{H} \varphi^{\prime}+\mathscr{B} \xi+\mathscr{H} \varphi=\lambda \xi, & x \in(0, l), \\ d_{3} \eta^{\prime \prime}-e w_{s j} \varphi+\left(s-2 r w_{s j}-e u_{s j}\right) \eta=\lambda \eta, & x \in(0, l), \\ \varphi^{\prime}=\xi^{\prime}=\eta^{\prime}=0, & x=0, l,\end{cases}
$$

where

$$
\begin{aligned}
P & =d_{4} h^{\prime}\left(u_{s j}\right) v_{s j}, \\
\mathscr{H} & =2 d_{4} h^{\prime}\left(u_{s j}\right) u_{s j}^{\prime}, \\
\mathscr{H} & =2 d_{4}\left(h^{\prime}\left(u_{s j}\right) v_{s j}^{\prime}+h^{\prime \prime}\left(u_{s j}\right) v_{s j} u_{s j}^{\prime}\right), \\
\mathscr{B} & =d_{4}\left(h^{\prime}\left(u_{s j}\right) u_{s j}^{\prime \prime}+h^{\prime \prime}\left(u_{s j}\right) u_{s j}^{\prime 2}\right)-u_{s j}-g, \\
\mathscr{H} & =d_{4}\left(h^{\prime}\left(u_{s j}\right) v_{s j}^{\prime \prime}+h^{\prime \prime}\left(u_{s j}\right) v_{s j} u_{s j}^{\prime \prime}+h^{\prime \prime}\left(u_{s j}\right) u_{s j}^{\prime 2} v_{s j}+2 h^{\prime \prime}\left(u_{s j}\right) u_{s j}^{\prime} v_{s j}^{\prime}\right)+b-v_{s j} .
\end{aligned}
$$

Setting 


$$
\begin{gathered}
\left\{\begin{array}{l}
\varphi=\varphi_{0}+\tau \varphi_{1}+\tau^{2} \varphi_{2}+\cdots, \\
\xi=\xi_{0}+\tau \xi_{1}+\cdots, \\
\eta=\eta_{0}+\tau \eta_{1}+\cdots, \\
\lambda=\lambda_{0}+\tau \lambda_{1}+\cdots,
\end{array}\right. \\
\left\{\begin{array}{l}
u_{s j}=\widetilde{u}+\tau u_{1}+\tau^{2} u_{2}+\cdots, \\
v_{s j}=\widetilde{v}+\tau v_{1}+\tau^{2} v_{2}+\cdots, \\
w_{s j}=\widetilde{w}+\tau w_{1}+\tau^{2} w_{2}+\cdots, \\
d_{4}=d_{4}^{0 j}+\tau d_{4}^{1 j}+\tau^{2} d_{4}^{2 j}+\cdots,
\end{array}\right.
\end{gathered}
$$

and substituting them into (38), we can obtain a system by equating the $O(1)$ terms

$$
\begin{cases}d_{1} \varphi_{0}^{\prime \prime}+(-1+a \widetilde{v}+\widetilde{w}) \varphi_{0}+(1+a \widetilde{u}) \xi_{0}+\widetilde{u} \eta_{0}=\lambda_{0} \varphi_{0}, & x \in(0, l), \\ \mathscr{L}_{3}^{j}\left(\xi_{0}, \varphi_{0}, \eta_{0}\right)=\lambda_{0} \xi_{0}-\lambda_{0} \frac{d_{4}^{0 j} h^{\prime}(\widetilde{u}) \widetilde{v}}{d_{1}} \varphi_{0}, & x \in(0, l), \\ d_{3} \eta_{0}^{\prime \prime}-e \widetilde{w} \varphi_{0}-r \widetilde{w} \eta_{0}=\lambda_{0} \eta_{0}, & x \in(0, l), \\ \varphi_{0}^{\prime}=\xi_{0}^{\prime}=\eta_{0}^{\prime}=0, & x=0, l,\end{cases}
$$

where

$$
\begin{aligned}
\mathscr{L}_{3}(\xi, \varphi, \eta):= & \left(d_{2}+d_{4}^{0} h(\widetilde{u})\right) \xi^{\prime \prime}-\left(g+\widetilde{u}+\frac{d_{4}^{0} h^{\prime}(\widetilde{u}) \widetilde{v}(1+a \widetilde{u})}{d_{1}}\right) \xi \\
& +\left(b-\widetilde{v}+\frac{d_{4}^{0} h^{\prime}(\widetilde{u}) \widetilde{v}^{2}}{d_{1} \widetilde{u}}\right) \varphi-\frac{d_{4}^{0} h^{\prime}(\widetilde{u}) \widetilde{u} \widetilde{v}}{d_{1}} \eta .
\end{aligned}
$$

It is obvious that the sign of $\lambda_{0}$ determines the stability of the stationary solution $\left(u_{s j}, v_{s j}, w_{s j}\right)$. To solve the eigenvalue problem (42), we can use $-\mu_{k}\left(\varphi_{0}, \xi_{0}, \eta_{0}\right)$ to replace $\left(\varphi_{0}^{\prime \prime}, \xi_{0}^{\prime \prime}, \eta_{0}^{\prime \prime}\right)$ for some integer $k \geq 0$. As to the existence of nonzero solution $\left(\varphi_{0}, \xi_{0}, \eta_{0}\right)$, we have

$$
\lambda_{0}^{3}+k_{1}(k) \lambda_{0}^{2}+k_{2}(k) \lambda_{0}+k_{3}(k)=0,
$$

$$
\begin{aligned}
& k_{1}(k)=\left(d_{1}+d_{2}+d_{3}+d_{4}^{0 j} h(\widetilde{u})\right) \mu_{k}+r \widetilde{w}+g+\widetilde{u}+\frac{\widetilde{v}}{\widetilde{u}}>0, \\
& k_{2}(k)=\left(\left(d_{1}+d_{3}\right) d_{4}^{0 j} h(\widetilde{u})+d_{1} d_{2}+d_{1} d_{3}+d_{2} d_{3}\right) \mu_{k}^{2} \\
& +\left\{(g+\widetilde{u}+r \widetilde{w}) d_{1}+\left(\frac{\widetilde{v}}{\widetilde{u}}+r \widetilde{w}\right) d_{2}+\left(\frac{\widetilde{v}}{\widetilde{u}}+g+\tilde{u}\right) d_{3}\right. \\
& \left.+\left(\left(\frac{\widetilde{v}}{\widetilde{u}}+r \widetilde{w}\right) h(\widetilde{u})+(1+a \tilde{u}) \widetilde{v} h^{\prime}(\widetilde{u})\right) d_{4}^{0 j}\right\} \mu_{k} \\
& +(g+\tilde{u}) r \tilde{w}+\tilde{u}+\widetilde{v}+(e-1) \tilde{u} \tilde{w}-b(1+a \tilde{u}) \\
& +\frac{(r \widetilde{w}+g) \widetilde{v}}{\widetilde{u}}-\frac{d^{0 j} h^{\prime}(\widetilde{u})}{d_{1} \tilde{u}}\left((\widetilde{v}+a \widetilde{u} \widetilde{v})^{2}+\widetilde{u} \widetilde{v}(\widetilde{w}-1)(1+a \widetilde{u})\right)
\end{aligned}
$$




$$
\begin{aligned}
& k_{3}(k)=\left(d_{1} d_{3} d_{4}^{0 j} h(\tilde{u})+d_{1} d_{2} d_{3}\right) \mu_{k}^{3}+\left\{\left(\left(\frac{\widetilde{v} d_{3}}{\widetilde{u}}+r \tilde{w} d_{1}\right) h(\widetilde{u})+\widetilde{v}(1+a \tilde{u}) d_{3} h^{\prime}(\widetilde{u})\right) d_{4}^{0 j}\right. \\
& \left.+r \widetilde{w} d_{2}+(g+\widetilde{u}) d_{1}+\frac{\widetilde{v}}{\bar{u}} d_{2} d_{3}\right\} \mu_{k}^{2}+\left(\frac{r \tilde{v} \widetilde{w}}{\widetilde{u}}+e \widetilde{u} \widetilde{w}\right) d_{4}^{0 j} h(\widetilde{u}) \\
& -\frac{d_{4}^{0 j} h^{\prime}(\widetilde{u})}{d_{1}}\left(-r \tilde{v} \widetilde{w} d_{1}(1+a \widetilde{u})+d_{3} \widetilde{v}(a \widetilde{v}(1+a \widetilde{u})+a \widetilde{u}(\widetilde{w}-1))\right)+r \widetilde{w}(g+\widetilde{u}) d_{1} \\
& \left.+\left(\frac{r \widetilde{v} \widetilde{w}}{\widetilde{u}}+e \widetilde{u} \widetilde{w}\right) d_{2}+\left(\frac{\widetilde{v} g}{\widetilde{u}}-b(1+a \widetilde{u})+\widetilde{u}+\widetilde{v}-\widetilde{u} \widetilde{w}\right) d_{3}\right\} \mu_{k} \\
& -\frac{d_{4}^{0 j} h^{\prime}(\widetilde{u}) a r \tilde{v} \widetilde{w}}{d_{1}}(\widetilde{v}(1+a \widetilde{u})+\widetilde{u}(\widetilde{w}-1))+\widetilde{w}\left(\frac{\widetilde{v} g r}{\widetilde{u}}-b r(1+a \widetilde{u})\right. \\
& +r(\widetilde{u}+\widetilde{v}-\widetilde{u} \widetilde{w})+e \widetilde{u}(g+\widetilde{u})) \\
& =d_{1} d_{2} d_{3} \mu_{k}^{3}+\left(r \widetilde{w} d_{1} d_{2}+(g+\widetilde{u}) d_{1} d_{3}+\frac{\widetilde{v}}{\widetilde{u}} d_{2} d_{3}\right) \mu_{k}^{2} \\
& +\left(r \tilde{w}(g+\widetilde{u}) d_{1}+\left(\frac{r \tilde{v} \widetilde{w}}{\widetilde{u}}+e \tilde{u} \widetilde{w}\right) d_{2}+\left(\frac{\widetilde{v} g}{\widetilde{u}}-b(1+a \tilde{u})+\widetilde{u}+\widetilde{v}-\tilde{u} \widetilde{w}\right) d_{3}\right) \mu_{k} \\
& +\widetilde{w}\left(\frac{\widetilde{v} g r}{\widetilde{u}}-b r(1+a \tilde{u})+r(\widetilde{u}+\widetilde{v}-\tilde{u} \widetilde{w})+e \widetilde{u}(g+\widetilde{u})\right)-d_{4}^{0 j}\left\{h ( \widetilde { u } ) \left(d_{1} d_{3} \mu_{k}^{3}\right.\right. \\
& \left.\left.+\left(r \widetilde{w} d_{1}+\frac{\widetilde{v}}{\widetilde{u}} d_{3}\right) \mu_{k}^{2}+\left(\frac{r \widetilde{w} \widetilde{v}}{\widetilde{u}}+e \widetilde{u} \widetilde{w}\right) \mu_{k}\right)+h^{\prime}(\widetilde{u}) \widetilde{v}(1+a \widetilde{u})\left(d_{3} \mu_{k}^{2}+r \widetilde{w} \mu_{k}\right)\right\} \\
& =\left\{h(\widetilde{u})\left(d_{1} d_{3} \mu_{k}^{3}+\left(r \widetilde{w} d_{1}+\frac{\widetilde{v}}{\widetilde{u}} d_{3}\right) \mu_{k}^{2}+\left(\frac{r \widetilde{w} \widetilde{v}}{\widetilde{u}}+e \widetilde{u} \widetilde{w}\right) \mu_{k}\right)\right. \\
& \left.+h^{\prime}(\widetilde{u}) \widetilde{v}(1+a \widetilde{u})\left(d_{3} \mu_{k}^{2}+r \widetilde{w} \mu_{k}\right)\right\}\left(d_{4}^{0 k}-d_{4}^{0 j}\right),
\end{aligned}
$$

and $d_{4}^{0 j}$ is given by (21).

It follows from the Routh-Hurwitz stability criteria that all the eigenvalues have negative real part $\left(\left(u_{s j}, v_{s j}, w_{s j}\right)\right.$ is locally asymptotically stable) if and only if $k_{3}(k)>0, k_{1}(k) k_{2}(k)-k_{3}(k)>0$ for all $k \in \mathbb{N}^{+}$. Hence, there exist some eigenvalues with positive real parts if one of the conditions above fails for some $k \in \mathbb{N}^{+}$.

We set $d_{4 \min }=d_{4}^{0 j_{0}}$ for a positive integer $j_{0}$ which is called the principle wave mode. This means that the first bifurcation will occur when the parameter $d_{4}$ crosses the bifurcation value $d_{4 \mathrm{~min}}$. Hence, $d_{4}^{0 j} \neq d_{4 \mathrm{~min}}$ if $j \neq j_{0}$ and there exists an integer $k=j_{0}$ such that $k_{3}(k)<0$. As such, equation (44) at least has a root with positive real part. Therefore, a necessary condition for the stability of $\left(u_{s j}, v_{s j}, w_{s j}\right)$ is stated as below.

Theorem 3 (stability criterion). If $j \neq j_{0}$, then $d_{4}^{0 j} \neq d_{4 \min }$ and the steady state $\left(u_{s j}, v_{s j}, w_{s j}\right)$ in (15) is unstable. In other words, if $\left(u_{s j}, v_{s j}, w_{s j}\right)$ is stable, then $j=j_{0}$.
Now, we determine the stability of the steady state $\left(u_{s j_{0}}, v_{s j_{0}}, w_{s j_{0}}\right)$. When $j=j_{0}$, we have $d_{4}^{0 j}=d_{4 \mathrm{~min}}$. Obviously, the principal eigenvalue for (42) is $\lambda_{0}=0$ with eigenvector:

$$
\begin{aligned}
\left(\varphi_{0}, \xi_{0}, \eta_{0}\right)= & \left(\omega_{j_{0}}, \frac{1}{1+a \tilde{u}}\left(\frac{\widetilde{v}}{\widetilde{u}}+\frac{e \tilde{u} \widetilde{w}}{r \widetilde{w}+d_{3} \mu_{j}}+d_{1} \mu_{j}\right) \omega_{j_{0}}\right. \\
& \left.-\frac{e \widetilde{w}}{r \widetilde{w}+d_{3} \mu_{j}} \omega_{j_{0}}\right) .
\end{aligned}
$$

In order to obtain the stability of $\left(u_{s j_{0}}, v_{s j_{0}}, w_{s j_{0}}\right)$, we need to evaluate $\lambda_{1}$ and $\lambda_{2}$. Again substituting (40) and (41) into (38) and equating the $O(\tau)$ terms, we have

$$
\begin{cases}d_{1} \varphi_{1}^{\prime \prime}+(-1+a \widetilde{v}+\widetilde{w}) \varphi_{1}+(1+a \widetilde{u}) \xi_{1}+\widetilde{u} \eta_{1}=\lambda_{1} \varphi_{0}+G_{1}, & x \in(0, l), \\ \mathscr{L}_{3}^{j}\left(\xi_{0}, \varphi_{0}, \eta_{0}\right)=\lambda_{1} \xi_{0}-\lambda_{1} \frac{d_{4}^{0 j} h^{\prime}(\widetilde{u}) \widetilde{v}}{d_{1}} \varphi_{0}+G_{2}, & x \in(0, l), \\ d_{3} \eta_{1}^{\prime \prime}-e \widetilde{w} \varphi_{1}-r \tilde{w} \eta_{1}=\lambda_{1} \eta_{0}+G_{3}, & x \in(0, l), \\ \varphi_{1}^{\prime}=\xi_{1}^{\prime}=\eta_{1}^{\prime}=0, & x=0, l,\end{cases}
$$


where

$$
\begin{aligned}
G_{1}= & -\left(a v_{1}+w_{1}\right) \varphi_{0}-a u_{1} \xi_{0}-u_{1} \eta_{0}, \\
G_{2}= & -d_{4}^{0 j}\left[h^{\prime}(\widetilde{u}) v_{1} \varphi_{0}+h^{\prime \prime}(\widetilde{u}) \widetilde{v} u_{1} \varphi_{0}+h^{\prime}(\widetilde{u}) u_{1} \xi_{0}\right]^{\prime \prime} \\
& +v_{1} \varphi_{0}+u_{1} \xi_{0}, \\
G_{3}= & e w_{1} \varphi_{0}+\left(2 r w_{1}+e u_{1}\right) \eta_{0} .
\end{aligned}
$$

The solvability condition for equation (47) gives

$$
\begin{aligned}
& \int_{0}^{l}\left(\lambda_{1} \varphi_{0}+G_{1}\right) \bar{u}_{2} \mathrm{~d} x+\int_{0}^{l}\left[\lambda_{1} \xi_{0}-\lambda_{1} \frac{d_{4}^{0 j} h^{\prime}(\widetilde{u}) \widetilde{v}}{d_{1}} \varphi_{0}+G_{2}\right] \bar{v}_{2} \mathrm{~d} x \\
& \quad+\int_{0}^{l}\left(\lambda_{1} \eta_{0}+G_{3}\right) \bar{w}_{2} \mathrm{~d} x=0,
\end{aligned}
$$

where $\left(\bar{u}_{2}, \bar{v}_{2}, \bar{w}_{2}\right)$ is given in (24) with $j=j_{0}$. Therefore,

$$
\lambda_{1}=-\frac{\int_{0}^{l}\left(G_{1} \bar{u}_{2}+G_{2} \bar{v}_{2}+G_{3} \bar{w}_{2}\right) \mathrm{d} x}{\int_{0}^{l}\left(\varphi_{0} \bar{u}_{2}+\left(\xi_{0}-\left(\left(d_{4}^{0 j} h^{\prime}(\widetilde{u}) \widetilde{v}\right) / d_{1}\right) \varphi_{0}\right) \bar{v}_{2}+\eta_{0} \bar{w}_{2}\right) \mathrm{d} x},
$$

where

$$
\begin{aligned}
& \int_{0}^{l}\left(\varphi_{0} \bar{u}_{2}+\left(\xi_{0}-\frac{d_{4}^{0 j} h^{\prime}(\widetilde{u}) \widetilde{v}}{d_{1}} \varphi_{0}\right) \bar{v}_{2}+\eta_{0} \bar{w}_{2}\right) \mathrm{d} x \\
& =\frac{1}{2} l\left\{\frac { 1 } { 1 + a \tilde { u } } \left(\left(d_{1}+d_{2}+d_{4}^{0 j_{0}} h(\widetilde{u})\right) \mu_{j_{0}}\right.\right. \\
& \left.\quad+\frac{e \widetilde{u} \widetilde{w}}{r \widetilde{w}+d_{3} \mu_{j_{0}}}+g+\widetilde{u}+\frac{\widetilde{v}}{\widetilde{u}}\right) \\
& \quad-\frac{d_{4}^{0 j_{0}} h^{\prime}(\widetilde{u}) \widetilde{v}}{d_{1}}-\frac{e \widetilde{w}}{\left(r \widetilde{w}+d_{3} \mu_{j_{0}}\right)^{2}(1+a \widetilde{u})} \\
& \left.\left(d_{2} \mu_{j}+d_{4}^{0} h(\widetilde{u}) \mu_{j_{0}}+g+\widetilde{u}\right)\right\} .
\end{aligned}
$$

A simple calculation gives

$$
\int_{0}^{l}\left(G_{1} \bar{u}_{2}+G_{2} \bar{v}_{2}+G_{3} \bar{w}_{2}\right) \mathrm{d} x=0 .
$$

Thus, $\lambda_{1}=0$. For this reason, we need further to compute $\lambda_{2}$. Since $G_{2}$ can be expressed as

$$
G_{2}=e_{1}+\left(4 d_{4}^{0} h^{\prime}(\widetilde{u}) e_{1} \mu_{j}+2 d_{4}^{0} h^{\prime \prime}(\widetilde{u}) \widetilde{v} \mu_{j}+e_{1}\right) \cos \left(2 \sqrt{\mu_{j}} x\right) \text {, }
$$

and we can find a particular solution $\left(\varphi_{1}, \xi_{1}, \eta_{1}\right)$ of (51) as

$$
\left\{\begin{array}{l}
\varphi_{1}=\bar{a}_{1}+\bar{a}_{2} \cos \left(2 \sqrt{\mu_{j_{0}}} x\right), \\
\xi_{1}=\bar{a}_{3}+\bar{a}_{4} \cos \left(2 \sqrt{\mu_{j_{0}}} x\right), \\
\eta_{1}=\bar{a}_{5}+\bar{a}_{6} \cos \left(2 \sqrt{\mu_{j_{0}}} x\right),
\end{array}\right.
$$

where

$$
\bar{a}_{i}=2 a_{i}\left(j_{0}\right), \quad i=1, \ldots, 6,
$$

with $a_{i}\left(j_{0}\right), i=1, \ldots, 6$, as defined in (30). A further similar computation by equating the $O\left(\tau^{2}\right)$ term gives the following system:

$$
\begin{cases}d_{1} \varphi_{2}^{\prime \prime}+(-1+a \widetilde{v}+\widetilde{w}) \varphi_{2}+(1+a \widetilde{u}) \xi_{2}+\widetilde{u} \eta_{2}=\lambda_{2} \varphi_{0}+G_{4}, & x \in(0, l), \\ \mathscr{L}_{3}^{j}\left(\xi_{0}, \varphi_{0}, \eta_{0}\right)=\lambda_{0} \xi_{0}-\lambda_{0} \frac{d_{4}^{0 j} h^{\prime}(\widetilde{u}) \widetilde{v}}{d_{1}} \varphi_{0}+G_{5}, & x \in(0, l), \\ d_{3} \eta_{2}^{\prime \prime}-e \widetilde{w} \varphi_{2}-r \widetilde{w} \eta_{2}=\lambda_{2} \eta_{0}+G_{6}, & x \in(0, l), \\ \varphi_{2}^{\prime}=\xi_{2}^{\prime}=\eta_{2}^{\prime}=0, & x=0, l,\end{cases}
$$

where 


$$
\begin{aligned}
G_{4}= & -\left(a v_{2}+w_{2}\right) \varphi_{0}-\left(a v_{1}+w_{1}\right) \varphi_{1}-a u_{2} \xi_{0}-a u_{1} \xi_{1}-u_{2} \eta_{0}-u_{1} \eta_{1}, \\
G_{5}= & -d_{4}^{0 j_{0}}\left\{h^{\prime}(\widetilde{u})\left[u_{1} \xi_{1}+v_{1} \varphi_{1}+u_{2} \xi_{0}+v_{2} \varphi_{0}\right]^{\prime \prime}\right. \\
& +h^{\prime \prime}(\widetilde{u})\left[\left(\widetilde{v} u_{1} \varphi_{1}+\widetilde{v} u_{2} \varphi_{0}+u_{1} v_{1} \varphi_{0}\right)^{\prime \prime}+\left(\frac{1}{2} u_{1}^{2} \xi_{0}^{\prime}+u_{1}^{\prime} u_{1} \xi_{0}\right)^{\prime \prime}\right] \\
& \left.+h^{\prime \prime \prime}(\widetilde{u}) \widetilde{v}\left(\frac{1}{2} u_{1}^{2} \varphi_{0}^{\prime}+u_{1}^{\prime} u_{1} \varphi_{0}\right)^{\prime}\right\}-d_{4}^{2 j_{0}}\left[h(\widetilde{u}) \xi_{0}^{\prime \prime}+h^{\prime}(\widetilde{u}) \widetilde{v} \varphi_{0}^{\prime \prime}\right] \\
& +u_{1} \xi_{1}+u_{2} \xi_{0}+v_{1} \varphi_{1}+v_{2} \varphi_{0}, \\
G_{6}= & e w_{2} \varphi_{0}+e w_{1} \varphi_{1}+\left(2 r w_{2}+e u_{2}\right) \eta_{0}+\left(2 r w_{1}+e u_{1}\right) \eta_{1} .
\end{aligned}
$$

Similarly, by applying the solvability condition of (56), we have

$$
\lambda_{2}=-\frac{\int_{0}^{l}\left(G_{4} \bar{u}_{2}+G_{5} \bar{v}_{2}+G_{6} \bar{w}_{2}\right) \mathrm{d} x}{\int_{0}^{l}\left(\varphi_{0} \bar{u}_{2}+\left(\xi_{0}-\left(\left(d_{4}^{0 j} h^{\prime}(\widetilde{u}) \widetilde{v}\right) / d_{1}\right) \varphi_{0}\right) \bar{v}_{2}+\eta_{0} \bar{w}_{2}\right) \mathrm{d} x} .
$$

A tedious computation leads to

$$
\int_{0}^{l}\left(G_{4} \bar{u}_{2}+G_{5} \bar{v}_{2}+G_{6} \bar{w}_{2}\right) \mathrm{d} x=-2 l \mu_{j_{0}} \Upsilon,
$$

with

$$
\Upsilon=\left[h(\widetilde{u}) e_{1}+h^{\prime}(\widetilde{u}) \widetilde{v}\right] d_{4}^{2 j_{0}} .
$$

Owing to the stability of nonconstant steady state, $\left(u_{s j_{0}}, v_{s j_{0}}, w_{s j_{0}}\right)$ depends on the sign of $\lambda_{2}$. Furthermore, $\Upsilon<0$ if $e_{1}<\left(2 \widetilde{u} \widetilde{v} / \varepsilon+\widetilde{u}^{2}\right)$, the denominator of (58) is positive since $e \widetilde{w}<\left(r \widetilde{w}+d_{3} \mu_{j_{0}}\right)^{2}(1+a \widetilde{u})$ and $\lambda_{2}<0$. Therefore, we have the following result.

Theorem 4. (stability). Let $j_{0}>0$ be a positive integer such that $d_{4}^{0 j_{0}}=d_{4 \min }$. Then, the small-amplitude steady state $\left(u_{s j}, v_{s j}, w_{s j}\right)$ is stable provided that $j=j_{0}$ and $\lambda_{2}<0$.

In particular, if $\max \left\{\left(e \widetilde{w} /\left(r \widetilde{w}+d_{3} \mu_{j_{0}}\right)^{2}\right),((\varepsilon+\right.$ $\left.\left.\left.\tilde{u}^{2}\right) / 2 \tilde{u} \widetilde{v}\right)\left((\tilde{v} / \tilde{u})+\left(e \tilde{u} \widetilde{w} /\left(r \tilde{w}+d_{3} \mu_{j_{0}}\right)\right)+d_{1} \mu_{j} \quad{ }_{0}\right)\right\}<1+a \tilde{u}$, then $\lambda_{2}<0$, and the small-amplitude steady state $\left(u_{s j_{0}}, v_{s j_{0}}, w_{s j_{0}}\right)$ is stable.

The following example can illustrate the result of Theorem 4 .

Example 1. For (1), in the interval $l=[0,10 \pi]$, set $a=1, b=$ $0.5, g=0.6, s=1.5, r=1.6, e=1, d_{1}=0.3, d_{2}=0.2, d$ ${ }_{3}=0.2$, and $\varepsilon=0.01$, and choose $d_{4}$ as the variable parameter. It is not hard to verify that the equilibrium point $\tilde{u}=(0.91,0.3,0.37), \quad d_{4}^{0 j_{0}}=6.02\left(j_{0}=9\right), \quad$ and $\max \{0.9$, $1.19\}<1.91$. From Theorem $4,\left(u_{s j}, v_{s j}, w_{s j}\right)$ is stable.

In conclusion, we study a predator-prey crossdiffusion model with cannibalism and discuss the effects of crossdiffusion on steady-state solutions of (1). We obtain the local structure of steady state bifurcation from the homogeneous steady states $(\widetilde{u}, \widetilde{v}, \widetilde{w})$ in one dimension by treating the crossdiffusion coefficient $d_{4}$ as a bifurcation parameter.
Moreover, we establish the stability criteria and find a selection mechanism of the principal eigenvalue. More specifically, if the stationary pattern is stable, then its principal wave mode $j$ is a positive integer $j_{0}$ at which $d_{4 j_{0}}$ is minimized. And all the rest bifurcations are unstable if $j \neq j_{0}$. In addition, the stable patterns can be derived if $j=j_{0}$ and $\lambda_{2}<0$.

\section{Data Availability}

The data used to support the findings of this study are available from the corresponding author upon request.

\section{Conflicts of Interest}

The authors declare that there are no conflicts of interest regarding the publication of this paper.

\section{Acknowledgments}

This research was supported by the National Natural Science Foundation of China (nos. 11761063 and 11661051).

\section{References}

[1] A. Jungel, Entropy Methods for Diffusive Partial Differential Equations, BCAM Springer Briefs, Springer, Berlin, Germany, 2016.

[2] L. Onsager and R. Fuoss, "Irreversible processes in electrolytes, diffusion, conductance and viscous flow in arbitrary mixtures of strong electrolytes," The Journal of Physical Chemistry, vol. 36, no. 11, pp. 2689-2778, 1932.

[3] R. L. Baldwin, P. J. Dunlop, and L. J. Gosting, "Interacting flows in liquid diffusion: equations for evaluation of the diffusion coefficients from moments of the refractive index gradient curves," Journal of the American Chemical Society, vol. 77, no. 20, pp. 5235-5238, 1955.

[4] S. M. Cox and I. M. Moroz, "Multiple bifurcations in triple convection with non-ideal boundary conditions," Physica D: Nonlinear Phenomena, vol. 93, no. 1-2, pp. 1-22, 1996.

[5] A. J. Pearlstein, R. M. Harris, and G. Terrones, "Cross-diffusion in reaction-diffusion models: analysis, numerics, and applications," in Progress in Industrial Mathematics at ECMI 2016, pp. 385-392, Springer, Berlin, Germany, 2017.

[6] K. R. Raghunatha, I. S. Shivakumara, and M. S. Swamy, "Effect of cross-diffusion on the stability of a triple-diffusive OldroydB fluid layer," Zeitschrift für Angewandte Mathematik und Physik, vol. 70, no. 4, p. 100, 2019.

[7] E. P. Zemskov, M. A. Tsyganov, and W. Horsthemke, "Multifront regime of a piecewise-linear FitzHugh-Nagumo model with cross diffusion," Physical Review E, vol. 99, no. 6, pp. 385-392, 2019.

[8] L. Chen, E. S. Daus, and A. Jungel, "Rigorous mean-field limit and cross-diffusion," Zeitschrift Fur Angewandte Mathematik Und Physik Zamp, vol. 70, no. 4, p. 122, 2019.

[9] P.-E. Druet and A. Jungel, "Analysis of cross-diffusion systems for fluid mixtures driven by a pressure gradient," SIAM Journal on Mathematical Analysis, vol. 52, no. 2, pp. 21792197, 2020.

[10] A. Jungel and M. Ptashnyk, "Homogenization of degenerate cross-diffusion systems," Journal of Differential Equations, vol. 267, no. 9, pp. 5543-5575, 2019.

[11] M. Benes and R. Stefan, "Global weak solutions for coupled transport processes in concrete walls at high temperatures," 
Zeitschrift Fur Angewandte Mathematik und Mechanik, vol. 93, no. 4, pp. 233-251, 2013.

[12] M. Benes, R. Stefan, and J. Zeman, "Analysis of coupled transport phenomena in concrete at elevated temperatures," Applied Mathematics and Computation, vol. 219, no. 13, pp. 7262-7274, 2013.

[13] N. Shigesada, K. Kawasaki, and E. Teramoto, "Spatial segregation of interacting species," Journal of Theoretical Biology, vol. 79, no. 1, pp. 83-99, 1979.

[14] B. Dubey, B. Das, and J. Hussain, "A predator-prey interaction model with self and cross-diffusion," Ecological Modelling, vol. 141, no. 1-3, pp. 67-76, 2001.

[15] M. E. Gurtin, "Some mathematical models for population dynamics that lead to segregation," Quarterly of Applied Mathematics, vol. 32, no. 1, pp. 1-9, 1974.

[16] J. B. Shukla and S. Verma, "Effects of convective and dispersive interactions on the stability of two species," Bulletin of Mathematical Biology, vol. 43, no. 5, pp. 593-610, 1981.

[17] Y. Lou and W.-M. Ni, "Diffusion, self-diffusion and crossdiffusion," Journal of Differential Equations, vol. 131, no. 1, pp. 79-131, 1996.

[18] K. Kuto and Y. Yamada, "Multiple coexistence states for a prey-predator system with cross-diffusion," Journal of Differential Equations, vol. 197, no. 2, pp. 315-348, 2004.

[19] K. Kuto, "Stability of steady-state solutions to a prey-predator system with cross-diffusion," Journal of Differential Equations, vol. 197, no. 2, pp. 293-314, 2004.

[20] P. Y. H. Pang and M. Wang, "Strategy and stationary pattern in a three-species predator-prey model," Journal of Differential Equations, vol. 200, no. 2, pp. 245-273, 2004.

[21] M. Ma, C. Ou, and Z.-A. Wang, "Stationary solutions of a volume-filling chemotaxis model with logistic growth and their stability," SIAM Journal on Applied Mathematics, vol. 72, no. 3, pp. 740-766, 2012.

[22] S. Fu, L. Zhang, and P. Hu, "Global behavior of solutions in a Lotka-Volterra predator-prey model with prey-stage structure," Nonlinear Analysis: Real World Applications, vol. 14, no. 5, pp. 2027-2045, 2013.

[23] K. G. Magnsson, "Destabilizing effect of cannibalism on a structured predator-prey system," Mathematical Biosciences, vol. 155, no. 1, pp. 61-75, 1999.

[24] J. M. Cushing, "A simple model of cannibalism," Mathematical Biosciences, vol. 107, no. 1, pp. 47-71, 1991.

[25] C. Kohlmeier and W. Ebenhöh, "The stabilizing role of cannibalism in a predator-prey system," Bulletin of Mathematical Biology, vol. 57, no. 3, pp. 401-411, 1995.

[26] L. Li and Z.-J. Wang, "Global stability of periodic solutions for a discrete predator-prey system with functional response," Nonlinear Dynamics, vol. 72, no. 3, pp. 507-516, 2013.

[27] A. Basheer, E. Quansah, S. Bhowmick, and R. D. Parshad, "Prey cannibalism alters the dynamics of Holling-Tanner-type predator-prey models," Nonlinear Dynamics, vol. 85, no. 4, pp. 2549-2567, 2016.

[28] F. Zhang, Y. Chen, and J. Li, "Dynamical analysis of a stagestructured predator-prey model with cannibalism," Mathematical Biosciences, vol. 307, pp. 33-41, 2019.

[29] L. R. Fox, "Cannibalism in natural populations," Annual Review of Ecology and Systematics, vol. 6, no. 1, pp. 87-106, 1975.

[30] B. Buonomo, D. Lacitignola, and S. Rionero, "Effect of prey growth and predator cannibalism rate on the stability of a structured population model," Nonlinear Analysis: Real World Applications, vol. 11, no. 2, pp. 1170-1181, 2010.
[31] F. V. D. Bosch and W. Gabriel, "Cannibalism in an age structured predator-prey system," Bulletin of Mathematical Biology, vol. 59, no. 3, pp. 551-567, 1997.

[32] C. Kaewmanee and I. M. Tang, "Cannibalism in an agestructured predator-prey system," Ecological Modelling, vol. 167, no. 3, pp. 213-220, 2003.

[33] R. Marlk and L. Pribylova, "An age-structured model of cannibalism," Electronic Journal of Differential Equations, vol. 106, pp. 285-296, 2006.

[34] S. Fu and X. Yang, "Nonconstant positive steady states of a predator-prey model with cannibalism," International Journal of Information and Systems Sciences, vol. 8, no. 2, pp. 250-260, 2012.

[35] A. A. Basheer, J. J. Lyu, and A. Giffin, "The "destabilizing" effect of cannibalism in a spatially explicit three-species age structured predator-prey model," Complexity, vol. 2017, Article ID 3896412, 15 pages, 2017.

[36] M. Ma and Z.-A. Wang, "Global bifurcation and stability of steady states for a reaction-diffusion-chemotaxis model with volume-filling effect," Nonlinearity, vol. 28, no. 8, pp. 2639-2660, 2015.

[37] M. G. Crandall and P. H. Rabinowitz, "Bifurcation from simple eigenvalues," Journal of Functional Analysis, vol. 8, no. 2, pp. 321-340, 1970.

[38] M. G. Crandall and P. H. Rabinowitz, "Bifurcation, perturbation of simple eigenvalues, itand linearized stability," Archive for Rational Mechanics and Analysis, vol. 52, no. 2, pp. 161-180, 1973. 